Bulletin of Pharmaceutical Sciences
Assiut University
Website: http://bpsa.journals.ekb.eg/
e-mail: bullpharm@aun.edu.eg

\title{
PERFORMANCE OF CURCUMIN IN NANOSIZED CARRIERS NIOSOMES AND ETHOSOMES AS POTENTIAL ANTI- INFLAMMATORY DELIVERY SYSTEM FOR TOPICAL APPLICATION
}

\author{
Mona M. El-Mahdy ${ }^{1 *}$, Abeer S. Hassan², Mahmoud El-Badry ${ }^{1,3}$ and Gamal El-Din A. El-Gindy ${ }^{1}$ \\ ${ }^{1}$ Department of Pharmaceutics, Faculty of Pharmacy, Assiut University. Assiut 71526, Egypt \\ ${ }^{2}$ Department of Pharmaceutics, Faculty of Pharmacy, South Valley University, Qena 83523, \\ Egypt \\ ${ }^{3}$ Assiut International Center of Nanomedicine, Al-Rajhy Liver Hospital, Assiut University, \\ Assiut 71515, Egypt
}

\begin{abstract}
Curcumin (CUR) is one of the most commonly used herbal product; it shows effective antiinflammatory and anti-oxidant effects. However, poor aqueous solubility and low permeability are the major challenges in therapeutic application of curcumin. One class of vesicular nanocarriers called "Niosome and ethosome" which have proved to possess distinct advantages were used to encapsulate curcumin and evaluated for their morphology, particle size, zeta potential, entrapment efficiency (EE\%) and drug release. They were incorporated into hydroxy propyl methyl cellulose (HPMC15000) gel then, evaluated on the rat skin via inhibition of carrageenan induced rat paw edema. The results showed that the particle size of curcumin loaded niosomes and ethosomes were ranged $(317.5 \pm 1.91$ to $558.3 \pm 8.587 \mathrm{~nm})$ and $(182.1 \pm 5.3$ to $354.5 \pm 30.03 \mathrm{~nm}$ ), respectively. Skin permeation studies demonstrated that CUR permeability coefficient through rat kin for gel formulations of loaded vesicles was $\sim$ four times higher as compared to free CUR. The in-vivo anti-inflammatory studies proved that gel formulations of CUR vesicles possessed higher significant inhibition of carrageenan induced rat paw edema when compared to pure curcumin. Accordingly, the results revealed that, curcumin loaded nanovesicels held great potential approaches as anti-inflammatory in topical application.
\end{abstract}

\section{INTRODUCTION}

Several natural products are widely used as medicines such as, quercetin, piperine, gingko and curcumin.

Curcumin (CUR), is unsaturated diketone (diferuloylmethane). It is one of the important active natural products that have been documented as medicinal agent. The medicinal efficacy of this product has been reported in Ayurvveda (Indian system of medicine) for 6000 years $^{1}$. It has wide range of potent biological and pharmacological actions, including anti-inflammatory, antioxidant, antifungal, anti-carcinogenic and wound healing activities ${ }^{2}$.
The pharmacological efficacy and safety (up to $12 \mathrm{~g} /$ day) of curcumin make it a potential compound for treatment and prevention of different human diseases ${ }^{3}$. However, poor aqueous solubility $(4-6 \mu \mathrm{g} / \mathrm{ml})$ in acidic and neutral $\mathrm{pH}$ with rapid metabolism in the body remain the main challenges for its therapeutic application ${ }^{4}$. In addition, it is susceptible to degradation in alkaline medium and when exposed to light ${ }^{5}$. Hence, water solubility of formulation combined with controlled release ability were desired for therapeutic application of curcumin ${ }^{6}$. Several attempts and new strategies were investigated to overcome all these shortcomings. Such methods include solid dispersions ${ }^{4}$,

Received in 29/3/2020 \& Accepted in 14/4/2020 
phospholipid complexes ${ }^{7}$, microspheres ${ }^{8}$, nanoemulsion ${ }^{9}$ and nanocrystals.

Self-assembly non-ionic surfactant-based vesicles (niosomes), have been widely used as drug nanocarriers. They are effective alternative to liposome due to their high stability, ease of preparation and low cost of surfactants which make them very attractive for several pharmaceutical and therapeutic applications. They have the potential to encapsulate both hydrophilic and lipophilic drug molecules because of the presence of both hydrophobic niosomal layer and hydrophilic core filled with water ${ }^{10}$.

They are able to enhance aqueous solubility as well as permeability of encapsulated active products through skin membrane ${ }^{11}$. Many drugs such as nimesulide ${ }^{12}$ and Diacerein ${ }^{13}$, have been successfully encapsulated in niosomes for topical application.

An ethosome is a novel vesicular carrier developed by Touitou et al., that exhibits improved skin delivery of drugs and identified with safety profiles for in-vitro and in-vivo efficacy. Ethosomes shows attractive properties as vesicular systems. They are composed of bilayer (aqueous and lipid) showing affinity for both hydrophilic and lipophilic drug leading to increased bioavailability. The delivery of lipophilic biological active products could be achieved at the targeted site along with protection of natural product present in the core material of lipids. Ethosomes are meant to increase the transdermal permeability of loaded drug as compared to liposomes or the ethanolic drug solution. Higher concentration of ethanol $(30-45 \%)$ is responsible for stearic stabilization, leading to deeper penetration of the loaded drug through the stratum corneum (SC) into the deeper skin layers with subsequent high transdermal flux.

Transdermal drug delivery system is considered as one of potential approach which can be used for active ingredient administration. It provides the controlled delivery of substance through ${ }^{14}$. Further it could not only act on the topical skin, but also deliver drugs to the blood circulation through skin.

The first attempt to incorporate nanotechnology for curcumin was by Tonnesen $^{15}$ where micellar solubilization of curcumin with a half-life of 2 months against hydrolytic reaction was observed.

Thangapazham et al. ${ }^{16}$ in another study demonstrated a better efficacy of nanoformulated curcumin in comparison to free curcumin. Their study incorporated the agent in liposomes coated with prostate-specific antibodies and demonstrated enhanced targeted delivery of curcumin. Treatment of cells with liposomal curcumin resulted in inhibition of cellular proliferation without affecting their viability with ten-fold dose advantage over free curcumin.

Another report demonstrated oral delivery of curcumin and suggested that the in-vitro release of curcumin was predominantly by diffusion phenomenon. In-vivo pharmacokinetics revealed that curcumin entrapped NPs demonstrate at least 9-fold increase in oral bioavailability when compared to curcumin administered with piperine as absorption enhancer ${ }^{17}$.

Choudhary et al. ${ }^{18}$ prepared curcumin liposomes for topical application. They showed that curcumin loaded liposomes provide localized permeation and deep penetration into the skin with higher encapsulation efficiency and better stability.

Another attempt was performed by Anwar et al. ${ }^{19}$. They prepared CUR nanoparticles with Tween 80 as a permeation enhancer and solubilizing agent by the supercritical antisolvent process.

Besides, CUR solid lipid nanoparticles for topical applications were prepared by Tiyaboonchai et $a l^{20}$. The obtained nanoparticles were stable and provided prolonged drug release. They investigated that SLNs could enhance CUR topical efficacy when compared with free CUR.

Rahman et al. ${ }^{18}$ formulated CUR niosomes using Span 60 and cholesterol to study the anti-inflammatory effect in rat model. They observed a better improvement in encapsulation efficiency (61\%) and a big particle size niosome $(1-5 \mu \mathrm{m})$.

Considering the above, the objective of this work was to investigate the performance of two different lipid-based vesicles (niosome and ethosome) as drug delivery systems of curcumin. Different formulations of CUR loaded niosomes and ethosomes were formulated, their vesicle size, morphological 
shape, encapsulation efficiency and in-vitro drug release were evaluated. The influence of different formulation variables on CUR entrapment efficiency was demonstrated. Further, the cytotoxicity effects of the selected niosome formulation of CUR will be conducted. The hydrogel formulation containing selected niosomes and ethosomes loaded with CUR were prepared to evaluate enhanced transdermal permeation through rat skin using in-vitro as well as in-vivo evaluations. Further, to indicate the efficacy of niosomes as a promising CUR nanocarrier, cytotoxicity study against hepatic tumor cells was performed.

\section{MATERIALS AND METHODS}

\section{Materials}

Curcumin (purity $>95 \%$ ) was purchased from (SD Fine-Chem limited Mumbai India). Hydroxyl propyl methylcellulose (HPMC15000), cholesterol and carrageenan were purchased from (Sigma Chemicals, St. Louis, MO, USA). Spectro/Por membranes (molecular weight cut-off $12-14 \mathrm{kDa}$ ) were purchased from Spectrum Medical Industries, Inc. (Laguna Hills, CA). Lecithin from soybean (AppliChem, Germany). Triton X-100 was purchased from (Park Scientific, Limited Northampton, UK). Chloroform, methanol, potassium dihydrogen phosphate, disodium hydrogen phosphate and sodium hydroxide were obtained from (United Company for Chem. and Med. Prep., Egypt)., $\operatorname{Span}^{\circledR} 20,40$, 60,80 , Tween $^{\circledR} 20,40,60,80$ and propylene glycol were purchased from (Adwic, El-Naser chemical co., Egypt). Dimethyl sulfoxide (DMSO), MTT and trypan blue dye were purchased from Sigma (St. Louis, Mo., USA). HepG-2 cells (human Hepatocellular cancer cell line), were obtained from the American Type Culture Collection (ATCC, Rockville, MD). All chemicals were of analytical grade and used as received.

\section{Methods}

\section{Preparation of drug loaded niosomes}

Niosomes containing curcumin were prepared using the thin film hydration method with different mixtures of nonionic surfactant/cholesterol with molar ratios of $1: 1$, $1: 2$ and 2:1. The composition of prepared niosomes vesicles are presented in table 1 . In brief, $500 \mu \mathrm{mol}$ of surfactants/cholesterol with drug were dissolved in chloroform : methanol $(2: 1 \mathrm{v} / \mathrm{v})$ mixture, in a round-bottom flask. The organic solvents were evaporated under vacuum at $60^{\circ} \mathrm{C}$ using rotary evaporator (Buchi 200, BU“ CHI Labortechnik AG, Flawil, Switzerland). The resulting thin lipid film produced hydrated with water, phosphate buffer ( $\mathrm{pH} \mathrm{5.5,6.5} \mathrm{and} \mathrm{7.4)} \mathrm{at} 60^{\circ} \mathrm{C}$ for 1 $\mathrm{hr}^{21 \& 22}$. The dispersion was left at $4^{\circ} \mathrm{C}$ for further study.

Table 1: Composition of different niosome formulations containing CUR.

\begin{tabular}{||l|c|c|c|c|c|c|c|c|c|c||}
\hline \multicolumn{1}{|c|}{ Formula } & N1 & N2 & N3 & N4 & N5 & N6 & N7 & N8 & N9 & N10 \\
\hline Curcumin $(\mathrm{mg})$ & 40 & 40 & 40 & 40 & 40 & 40 & 40 & 40 & 40 & 40 \\
\hline Cholesterol $(\mu \mathrm{mol})$ & 250 & 250 & 250 & 250 & 250 & 250 & 250 & 250 & - & - \\
\hline Span $20(\mu \mathrm{mol})$ & 125 & - & - & - & - & - & - & - & - & - \\
\hline Span 40 $(\mu \mathrm{mol})$ & - & 125 & - & - & - & - & - & - & - & - \\
\hline Span 60 $(\mu \mathrm{mol})$ & - & - & 125 & - & - & - & - & 125 & 250 & - \\
\hline Span $80(\mu \mathrm{mol})$ & - & - & - & 125 & - & - & - & - & - & - \\
\hline Tween 20 $(\mu \mathrm{mol})$ & 125 & - & - & - & - & - & - & - & - & - \\
\hline Tween 40 $(\mu \mathrm{mol})$ & - & 125 & - & - & 125 & 125 & 125 & - & - & - \\
\hline Tween 60 $(\mu \mathrm{mol})$ & - & - & 125 & - & - & - & - & - & - & 250 \\
\hline Tween 80 $(\mu \mathrm{mol})$ & - & - & - & 125 & - & - & - & 125 & - & - \\
\hline GMS $(\mu \mathrm{mol})$ & - & - & - & - & 125 & - & - & - & - & - \\
\hline Myrj 52 $(\mu \mathrm{mol})$ & - & - & - & - & - & 125 & - & - & - & - \\
\hline Brij 35 $(\mu \mathrm{mol})$ & - & - & - & - & - & - & 125 & - & - & - \\
\hline Niosome formation & F & F & F & NF & F & NF & NF & F & NF & NF \\
\hline
\end{tabular}

CUR, curcumin, F, formed; NF, not formed. 


\section{Preparation of drug loaded ethosomes}

Curcumin ethosomes were prepared using ethanol injection method. In brief, lecithin, cholesterol and curcumin were dissolved in anhydrous ethanol in a sealed glass container. The solution was stirred on magnetic stirrer (Gallenkamp, Loughborough, UK) at $30^{\circ} \mathrm{C}$ for $15 \mathrm{~min}$. Distilled water was added gradually at a constant rate of $100 \mu \mathrm{l} / \mathrm{min}$. The ethosomes dispersion was left at $4^{\circ} \mathrm{C}$ for further study.

\section{Characterization of vesicular nano-carriers formulations}

1- Microscopic examination

Small amount of the prepared CUR niosomes and ethosomes was spread on a glass slide and examined microscopically at magnification of $1000 \mathrm{x}$ using a binocular microscope equipped with a camera (Motic, Japan).

\section{2- Transmission electron microscopy (TEM)}

The niosomal dispersion of CUR was diluted 10 -folds using distilled water. A drop of diluted CUR vesicle dispersion was applied to a carbon coated 300 mesh copper grid and left for $1 \mathrm{~min}$ to allow some of vesicles to adhere to the carbon substrate. Excess dispersion was removed by a piece of filter paper followed by rising the grid twice in deionized water for 3-5 s. Next, a drop of $2 \%$ aqueous solution of uranyl acetate was applied for $1 \mathrm{~s}$. The remaining solution was removed by absorbing the liquid with the tip of a piece of filter paper and the sample was air dried. Afterward, the sample was viewed under the microscope at 10-100 k magnification power using an accelerating voltage of $100 \mathrm{kV}$ using the JEOL TEM (Model 100 CX II; Tokyo, Japan).

\section{3- Dynamic light scattering (DLS) and Zeta- Potential measurements}

The mean hydrodynamic diameters and size distribution (polydispersity indices, PDIs) of some CUR niosomes and ethosomes in water were measured using a Zetasizer Nano ZS instrument (Malvern Instruments, Malvern, UK) equipped with a backscattered light detector operating at $173^{\circ}$. The CONTIN program was used to extract size distributions from the autocorrelation functions. The zetapotential values were determined by laser
Doppler anemometry using a Malvern Zetasizer Nanoseries ZS.

\section{4- Encapsulation efficiency (EE\%)}

Niosomes and ethosomes containing CUR were separated from un-entrapped drug by cooling centrifugation at $14000 \mathrm{rpm}$ (Centurion Scientific Ltd., W. Sussex, U.K) for $60 \mathrm{~min}$ at $4^{\circ} \mathrm{C}^{23}$. After centrifugation, the formed niosomal and ethosomal pellets were washed and recentrifuged the supernatant was removed carefully to separate the non-encapsulated curcumin. One milliliter of the supernatant was diluted to $10 \mathrm{ml}$ with methanol, and the amount of drug was determined spectrophotometrically at $\lambda_{\max } 430 \mathrm{~nm}$ (Jenway Model 6305, U.K) ${ }^{18}$.

The entrapment efficiency was calculated by the following equation ${ }^{24}$.

$$
E E(\%)=\frac{(T-C)}{T} \times 100
$$

Where $T$ is the total amount of CUR added, and $\mathrm{C}$ is the amount of CUR in the supernatant.

Each experiment was performed in triplicate.

\section{Preparation of CUR gel formulations}

The selected curcumin-loaded niosomes and ethosomes (equivalent to $0.5 \% \mathrm{w} / \mathrm{w}$ drug) were incorporated into gel base (3.0\% HPMC 15000). Gels containing free curcumin was prepared by incorporated of CUR solution into gel by stirring until a homogenous niosomal and ethosomal gel formulations were obtained.

\section{In-vitro drug release through cellophane membrane}

In-vitro drug release of CUR from niosomes and ethosomes was investigated using cellophane membrane dialysis tubing. In brief, $1 \mathrm{ml}$ sample of niosome and ethosome was placed on a semipermeable cellophane membrane formerly immersed in phosphate buffer of pH 5.5 for $24 \mathrm{hrs}$ then was struggling over the lower open end of a glass tube made watertight by a rubber band. Tubes was suspended in a beaker (receptor compartment) containing $100 \mathrm{ml}$ of the release medium composed of phosphate buffer $\mathrm{pH} 5.5$ and Tween ${ }^{\circledR} 80(0.5 \%)$ to maintain sink condition. The system was maintained for $24 \mathrm{hrs}$ at $37^{\circ} \mathrm{C}$ in a thermostatically controlled shaker water bath at $50 \mathrm{rpm}$ (Gesellschaft Labor Technik M.B.H.\&Co., GFL. Germany). Samples of $5 \mathrm{ml}$ were withdrawn at intervals of $0.5,1,2,3,4,6$, 
8,12 , and $24 \mathrm{~h}$. The volume of each withdrawn sample was replaced by the same volume of same dissolution medium maintained at the same temperature to keep constant volume and maintain sink condition. Also, the release of CUR selected gel formulations was determined. Samples were analyzed for curcumin content spectrophotometrically at $\lambda_{\max } 430 \mathrm{~nm}$ against blank similarly treated. Each experiment was repeated in triplicate.

\section{Kinetic release study}

The data obtained from the in-vitro release studies was analyzed using linear regression method $\left(\mathrm{r}^{2}\right)$. The release data analysis was studied according to zero order kinetic model, Higuchi diffusion model and KorsmeyerPeppas model).

\section{Zero order kinetics}

$$
\mathrm{Q}=\mathrm{k}_{0} \mathrm{t}^{25}
$$

Where $\mathrm{Q}$ is the drug released at time $\mathrm{t}$.

$\mathrm{K}_{0}$ is the zero order release constant.

$\mathrm{t}$ is the time.

\section{Higuchi model}

$$
\mathrm{Q}=\mathrm{k}_{\mathrm{H}} \mathrm{t}^{1 / 226}
$$

Where $\mathrm{Q}$ is the amount of drug released at time $t$ per unit area.

$\mathrm{K}_{\mathrm{H}}$ is the Higuchi release rate constant.

\section{Korsmeyer-Peppas equation}

$$
\mathrm{M}_{\mathrm{t}} / \mathrm{M}_{\infty}=\mathrm{kt}^{\mathrm{n}} 27
$$

Where $M_{t} / M_{\infty}$ is the fraction of drug released at time $\mathrm{t}$.

$\mathrm{n}$ is the release exponent.

$\mathrm{n}$ value is indicative for the drug release mechanism where $\mathrm{n} \leq 0.5$ indicates a fickian diffusion mechanism, while $0.5<\mathrm{n}<1$ indicates a non-fickian mechanism (anomalous diffusion). If $n=1$, it indicates a zero order mechanism (case II relaxation). In case of $n>1$, it indicates a super case II transport.

Anomalous diffusion or non-fickian diffusion refers to combination of both diffusion and erosion controlled release rate while case II relaxation and super case II transport refer to erosion of the polymeric matrix.
Ex-vivo permeability and skin deposition studies

\section{1- Ex-vivo permeability}

Ex-vivo permeability of CUR through rat hairless skin was studied using optimum samples of CUR niosomes, ethosomes and gel formulations. Male Wister rats weighing 200$250 \mathrm{~g}$ were used in this study. Experimental procedures were in accordance with the guidelines of the Animal Ethics Committee of Faculty of Pharmacy, Assiut University, Egypt (Approval no. S8-19/2019). Rats were sacrificed immediately before the start of the experiment. A full thickness skin was excised from abdominal site then was washed with water. The skin was washed with phosphate buffer (pH 5.5). The skin samples were firmly stretched using rubber bands over one end of glass tubes opened from both sides and having an internal diameter of $2.4 \mathrm{~cm}$. The stratum corneum was facing upwards (donor side) and the dermal surface was facing downwards and allowed to be in contact with receptor compartment. The tubes were immersed in a beaker containing $100 \mathrm{~mL}$ of phosphate buffer pH 5.5 (receptor compartment). The beakers were placed into a shaking water bath maintained at $37 \pm 0.5^{\circ} \mathrm{C}$ and $50 \mathrm{rpm}$. Samples of $5 \mathrm{~mL}$ were withdrawn from the receptor compartment at time intervals of $0.5,1,2,3,4$ and $6 \mathrm{~h}$. and were replaced by the same volume of fresh phosphate buffer. Samples were analyzed for CUR content spectrophotometrically at $\lambda_{\max } 430 \mathrm{~nm}$ against a similarly treated blank. These experiments were done in triplicates and the mean \pm SD were calculated. The cumulative percent of curcumin, which permeated to the rat skin, was plotted against time. The transdermal drug flux was calculated from the slope of the linear portion of the plot.

\section{2- Calculation of apparent cur permeability coefficients $\left(\mathbf{P}_{\text {app }}\right)$}

The apparent permeability coefficients $\left(\mathrm{P}_{\text {app }}, \mathrm{cm} / \mathrm{s}\right)$ of CUR were calculated using Eq. (2):

$$
\text { Papp }=\frac{1}{A C_{0}} \times \frac{d Q}{d t}
$$

where $\mathrm{dQ} / \mathrm{dt}$ is the rate of appearance of CUR in the receptor compartment $(\mathrm{nmol} / \mathrm{s}), \mathrm{A}$ is the surface area of the skin, and $\mathrm{C}_{0}$ is the initial CUR concentration (nM) in the tested sample at $\mathrm{t}=0$. Permeability rates $(\mathrm{dQ} / \mathrm{dt})$ were 
obtained from the permeation profiles of each tested sample. The regression coefficients $\left(\mathrm{r}^{2}\right)$ obtained from the curve were generally between 0.80 and 0.99 .

\section{3- Drug retention studies in skin}

This study was investigated to determine the amount of CUR deposited in the skin. In brief, after completion of the skin permeation experiment (6 hrs), the side of the skin exposed to donor compartment (containing formulation) was washed with phosphate buffer $\mathrm{pH} 5.5$, to remove any adhered remaining drug from the skin surface. The skin was then cut into small pieces and shaken in 50\% methanol $(10 \mathrm{ml})$ for 24 hrs to extract CUR into the medium. At the end of $24 \mathrm{hrs}$ the medium was filtered and analyzed spectrophotometric at $\lambda_{\max } 430 \mathrm{~nm}$ for CUR content.

\section{In-vivo anti-inflammatory study (carrageenan paw edema inflammation model)}

In-vivo anti-inflammatory activity of CUR selected formulations was performed based on the inhibition of the hind paw edema in rats. Experimental procedures were in accordance with the guidelines of the Animal Ethics Committee of Faculty of Pharmacy Assiut University, Egypt.

Male albino twenty-five rats weighing about $200 \mathrm{~g}$ were used. All the rats were kept under standard laboratory conditions at temperature of $25^{\circ} \mathrm{C}$ and relative humidity of $55 \%$. The rats were housed in polypropylene cages (five rats per cage) with free access to a standard laboratory diet (lipton feed) and water ad libitum. Thirty minutes before the application of each formulation, the thickness of the hind paws was measured by means of a Vernier Caliper (SMEC, China). $1 \%$ w/v carrageenan solution in normal saline was injected subcutaneously into the right hind paw to induce inflammation. The animals were divided into five groups, each group comprised of five animals. The animals of group 1 received only carrageenan (control), groups 2 and 3 received HPMC 15000 gel containing CUR entrapped niosome (N3 G), and CUR ethosomes (E9 G), respectively. Group 4 received CUR (free drug) HPMC 15000 gel and group 5 received anti-inflammatory commercial drug (Nonsteroidal anti- inflammatory ketoprofen: Fastum ${ }^{\circledR}$ gel,). After half an hour, $0.5 \mathrm{~g}$ of each gel was applied to the same right hind paw (except the control group). The increase in paw thickness was measured before carrageenan injection and immediately after carrageenan injection (zero time) and then every hour for up to $8 \mathrm{hrs}$ using a Vernier Caliper. The percentage swelling of the paw of edema was calculated by employing the following equation ${ }^{28}$ The data were reported as mean $\pm \mathrm{SD}(n=5)$.

$$
\% \text { Swelling }=\frac{\left(V_{t}-V_{i}\right)}{V_{i}} \times 100
$$

Where: $V_{t}$ is the paw thickness at each time interval, and Vi the initial paw thickness before carrageenan injection. The average paw swelling in CUR treated rats were compared with that of control rats (which received placebo) and the percent inhibition of paw edema was estimates using the following equation:

$\%$ Inhibition $=$

[1 - (\% swelling treated / \% swelling control $)] \times 100$

Where swelling treated is the mean value observed in the treated group, and swelling control is the mean value observed in the control group.

\section{Stability studies}

Stability studies were carried out for the selected formulations of two types of vesicles (niosomes and ethosomes). They were kept at room temperature for three months and in refrigerator for six months. Physical evaluation of the samples stability was carried out by visual inspection, determination of mean vesicle size and size distribution. Further, chemical stability was evaluated by determination of encapsulation efficiency by spectrophotometer and drug degradation by thin layer chromatography (TLC).

\section{Thin layer chromatography (TLC)}

Stability of CUR in the selected niosomal (N3) and ethosomal (E9) dispersions was studied by TLC. Also, stability of free CUR in aqueous suspension, alcoholic and hydroalcoholic solution was studied under the same conditions. Triton (10\%) was added to the lipid vesicles before spotting on the TLC for $1 \mathrm{~min}$ to allow for the lysis of niosomes and 
ethosomes and release of the entrapped drugs to be available for detection. TLC analyses were performed on $7 \mathrm{~cm} \times 20 \mathrm{~cm}$ aluminum plates coated with $0.2 \mathrm{~mm}$ layer of silica gel (60 F254). Samples were loaded onto the plate $10 \mathrm{~mm}$ from the bottom and $10 \mathrm{~mm}$ from the side edges of the plate with a band length of 4 $\mathrm{mm}$. Samples were spotted using linomat V semiautomatic spotting device under continuous drying stream of nitrogen gas. Linear ascending development with chloroform: methanol $(75: 5 \mathrm{v} / \mathrm{v})$ as a mobile phase was performed in a glass chamber previously saturated with the mobile phase for $15 \mathrm{~min}$ at room temperature $\left(25 \pm 1^{\circ} \mathrm{C}\right)$. The optimized development distance and development time were $60 \mathrm{~mm}$ and $10 \mathrm{~min}$, respectively. After development, the plates were dried completely and the spots were scanned densitometrically by Camag TLC scanner III (Camag, Muttenz, Switzerland). The TLC scanner was adjusted at a wavelength of $\lambda_{\max } 430 \mathrm{~nm}$. All measurements were analyzed by winCATS software. Retention factor (Rf) values, concentrations of the separated compounds and appearance of new spots were determined. All measurements were performed in triplicates.

\section{Statistical analysis}

The differences between the mean values were analyzed using Graph Pad Prism software version 5. One-way analysis of variance (ANOVA) was used to analyze the differences between experimental groups. A probability of less than $0.05 \quad(p<0.05)$ was considered statistically significant. All experiments were done in triplicates and the results are presented as mean $\pm \mathrm{SD}$.

\section{RESULTS AND DISCUSSION}

\section{Preparation of CUR niosomes}

CUR is a natural product has various medical benefits. One of the challenges is to enhance the water solubility of drug at site of action and increase the permeation of the drug to the systemic circulation. CUR-loaded niosomes composed of $500 \mu \mathrm{mol}$ total lipids were prepared by classical thin film hydration method using different ratios (1:1, 1:2 and 2:1) of cholesterol $(\mathrm{CH})$ and non-ionic surfactants (NIS). Equal molarity of non-ionic surfactants and cholesterol was selected for further preparations. This may improve bilayer compactness and enhance the entrapment efficiency ${ }^{29}$. The niosome formation ability of the studied surfactants is summarized in table 1 .

\section{Characterization of CUR niosomes 1- Transmission electron microscopy (TEM)}

The presence of vesicles in niosomal dispersion was indicated by observing the niosomal dispersion after $24 \mathrm{hrs}$ using an optical microscope. The lipid vesicles are unilamellar and spherical. This indicated by TEM (Fig. 1). These results were previously obtained in niosomal vesicles of carvedilol ${ }^{30}$.

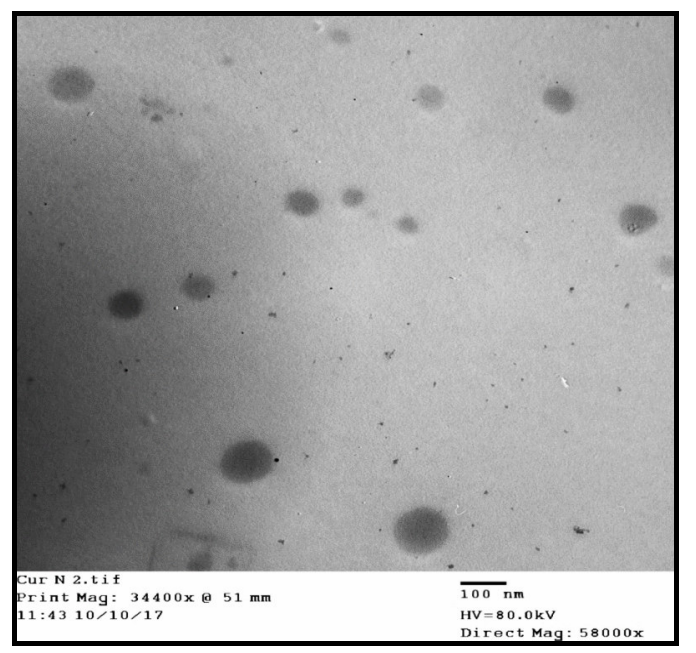

Fig. 1: Transmission electron microscopy micrograph of curcumin niosomes.

\section{2- Particle size and Zeta potential measurements}

The particle size, PDI and zeta potential of CUR freshly prepared niosomes are illustrated in table 2. The size of vesicles in the range of $317.5 \pm 1.91$ to $558.3 \pm 8.587 \mathrm{~nm}$ with PDI ranging from $0.426 \pm 0.024$ to $0.599 \pm 0.106$ indicating a relatively uniform homogenous particle size distribution ${ }^{30}$. The prepared niosomes of Span60-Tween 60 show the lowest value of PDI as $0.426 \pm 0.024$ which indicates a narrow distribution of niosomes size. Also, niosomes of Span 60 -Tween 60 shows particle size smaller than that obtained from other surfactants. The zeta potential values for the prepared curcumin loaded niosomes are presented in table 2. It is noticed that CUR 
niosomes exhibits higher negative $\mathrm{ZP}$ values $(-39.1 \pm 0.666 \mathrm{mV}$ and $-29.0 \pm 2.15 \mathrm{mV}$ for $\mathrm{N} 3$ and N5, respectively), indicating higher stability. The prepared niosomes in this study showed smaller particle size as compared with previous work ${ }^{18}$. The authors found that CUR loaded niosomes have large particle size CUR (1-5 $\mu \mathrm{m})$.

\section{Effect of different parameters on CUR niosomes encapsulation efficiency \\ 1- Effect of surfactant structure}

As shown in table 2, the rank order of encapsulation efficiency using different nonionic surfactants is in the following order N3 $\left(\operatorname{Span}^{\circledR}{ }^{\circledR}\right.$ 60-Tween $\left.{ }^{\circledR} 60\right)>\mathrm{N} 2{ }$ Span $^{\circledR} 40$ Tween $\left.^{\circledR} 40\right)>$ N5 (GMS-Tween $\left.{ }^{\circledR} 40\right)>$ N8 $\left(\operatorname{Span}^{\circledR}\right.$ 60-Tween $\left.{ }^{\circledR} 80\right)>\mathrm{N} 1 \quad\left(\operatorname{Span}^{\circledR} 20-\right.$ Tween ${ }^{\circledR}$ 20). It is noticed that niosome of Span $^{\circledR}$ 60-Tween 60 (N3) has significantly higher entrapment efficiency (89\%) than other mixtures $(p<0.05)$. This is due to the surfactant chemical structure and can be explained by numerous facts: a) $\operatorname{Span}^{\circledR} 60$ and Tween ${ }^{\circledR} 60$ have relatively long and saturated paraffin chain (stearyl C18), b) The large hydrophilic head group of Tween 60 (polyoxyethylene groups) which could solubilize CUR due to the presence of phenolic groups in CUR structure, suggesting the formation of hydrogen bond ${ }^{31}$.

Another notable finding is that niosomes prepared using Tween 60 and 40 show higher EE\% compared to those prepared using Tween ${ }^{\circledR} 80$ (POE (20) sorbitan monooleate), this may be attributed to the presence of unsaturated double bond in paraffin chain of Tween 80 and the introduction of double bonds made the chains bend, that may lead to the formation of a leaky and permeable niosomal membrane $^{32}$. On the other hand, Brij ${ }^{\circledR} 35$ (lauryl C12 chain surfactants) and $\mathrm{Myrj}^{\circledR} 52$ (polyoxyethylen 40 stearate) could not form lipid vesicle by thin film hydration. This may be because of the smaller critical packing parameter (CPP) and higher HLB values (16 and 16.9 for $\mathrm{Brij}^{\circledR} 35$ and $\mathrm{Myrj}^{\circledR}$ 52, respectively) which indicates low lipophilicity in comparison with hydrophilic surface area of these surfactant molecules ${ }^{33}$.

\section{2- Effect of CUR concentration}

The effect of increasing CUR concentration on encapsulation efficiency EE\% in the selected niosomal formation (N3) is presented in figure 2 . The niosomes of 5,10, 20, 30, 40, 50 and $100 \mathrm{mg}$ CUR were performed of $500 \mu$ mole total amount of lipid. The results depict that, the encapsulation increases (from $43 \%$ to $89 \%$ ) on increasing the drug concentration (from 5 to $40 \mathrm{mg} / 10 \mathrm{ml}$ ), respectively. However, from the statistical analysis on view, it is found that, the differences between encapsulation efficiency on increasing drug amount from 5 to $40 \mathrm{mg}$ in the formulation are significant $(p<0.05)$. This may be due to the saturation of the hydration medium with curcumin which forces the drug to be entrapped into lipid vesicles.

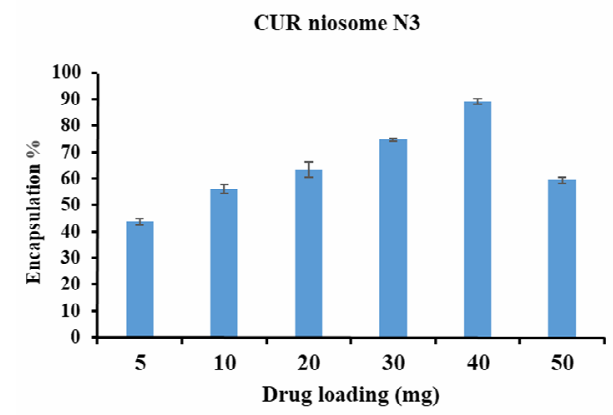

Fig. 2: Effect of CUR loading on niosomal encapsulation efficiency. All results are expressed in mean $\pm \operatorname{SD}(n=3)$.

Table 2: Particle size, polydispersity index, zeta potential and encapsulation efficiency of the formed CUR niosome formulations. Each result is the mean value $\pm \operatorname{SD}(n=3)$.

\begin{tabular}{|c|c|c|c|c||}
\hline Formulation & $\begin{array}{c}\text { Particle size } \\
(\mathrm{nm})\end{array}$ & PDI & $\begin{array}{c}\text { Zeta potential } \\
(\mathrm{mV})\end{array}$ & EE\% \\
\hline $\mathrm{N} 1$ & $558.3 \pm 8.58$ & $0.599 \pm 0.10$ & $-26.4 \pm 0.15$ & 17 \\
\hline $\mathrm{N} 2$ & $420.21 \pm 10.32$ & $0.543 \pm 0.04$ & $-22.7 \pm 0.29$ & 77 \\
\hline $\mathrm{N} 3$ & $317.5 \pm 1.91$ & $0.426 \pm 0.05$ & $-39.1 \pm 0.66$ & 89 \\
\hline $\mathrm{N} 5$ & $373.0 \pm 16.38$ & $0.596 \pm 0.09$ & $-29.0 \pm 2.15$ & 65 \\
\hline $\mathrm{N} 8$ & $382.9 \pm 7.75$ & $0.491 \pm 0.08$ & $-24.2 \pm 2.15$ & 40 \\
\hline Blank of N3 & $776.7 \pm 8.18$ & $0.573 \pm 0.08$ & $-22.7 \pm 0.29$ & - \\
\hline
\end{tabular}

CUR, curcumin, SD, standard deviation, EE, encapsulation efficiency, PDI, polydispersity index. 


\section{Preparation of CUR ethosomes}

Ethosomes are vesicular systems with hydrated lipid bilayers. The composition of different ethosomal formulations is summarized in table 3. Formulations containing different lecithin percentage and different drug amounts to determine the effect on the particle size, entrapment efficiency and drug release. Cholesterol $(20 \mathrm{mg}$ ) was added to increase the rigidity of membrane bilayer and stability of ethosomal vesicles. The results revealed that, the ethosomal formulations that have more than $40 \mathrm{mg}$ CUR were not formed and drug precipitation was observed during preparation. This might be due to limited CUR solubility and saturation of lipid bilayer of ethosmes above this amount of drug.

\section{Size, Shape and Zeta potential of CUR ethosomes}

Figure 3 shows the unilamellar spherical and regular vesicles. Ethosomes show a narrow particle size distribution (from $0.427 \pm 0.091$ to
$0.540 \pm 0.048$ ) (Table 4). The size of plain ethosome is $110.77 \pm 0.81$ and it is significantly increased by increasing the drug loading $(p<$ $0.05)$. These results are in agreement with the previously reported for paclitaxel ethosomes ${ }^{32}$ and explained by incorporation the drug into the lipid bilayer and this might lead to enlargement of lipid bilayer to accommodate the entrapped drug. The results in table 4 show that, minimum vesicle size is $182.1 \pm 5.3 \mathrm{~nm}$ (E1) whereas maximum is $354.5 \pm 30.03 \mathrm{~nm}$ (E9), it is evident that, the size of the vesicles is dependent on the concentration of soya lecithin which increased on increasing concentrations of soya lecithin from 1-3\% w/v.

Zeta-potential of CUR ethosomes is ranging from $-43 \pm 1.6$ to $-64.32 \pm 2.6$ (Table 4). This negative charge may be attributed to the effect of lecithin (phospholipids) and ethanol ${ }^{34}$. Formulation E9 has significantly highest negative zeta potential $(p<0.05)$ which may predict good stability without any signs of aggregation and precipitation.

Table 3: Composition and encapsulation of different ethosome formulations containing CUR.

\begin{tabular}{|c|c|c|c|c|c|c|}
\hline Formula & $\begin{array}{c}\text { Drug } \\
(\mathrm{mg})\end{array}$ & $\begin{array}{c}\text { Lecithin } \\
\%\end{array}$ & $\begin{array}{c}\text { Cholesterol } \\
(\mathrm{mg})\end{array}$ & $\begin{array}{c}\text { Ethanol } \\
(\mathrm{ml})\end{array}$ & $\begin{array}{c}\text { Water } \\
(\mathrm{ml})\end{array}$ & EE\% \\
\hline E1 & 10 & 1 & 20 & 3 & 7 & $80.34 \pm 0.54$ \\
\hline E2 & 10 & 2 & 20 & 3 & 7 & $82.34 \pm 0.14$ \\
\hline E3 & 10 & 3 & 20 & 3 & 7 & $84.87 \pm 0.98$ \\
\hline E4 & 20 & 1 & 20 & 3 & 7 & $85.87 \pm 1.76$ \\
\hline E5 & 20 & 2 & 20 & 3 & 7 & $86.54 \pm 2.32$ \\
\hline E6 & 20 & 3 & 20 & 3 & 7 & $89.54 \pm 0.95$ \\
\hline E7 & 30 & 1 & 20 & 3 & 7 & $90.53 \pm 0.87$ \\
\hline E8 & 30 & 2 & 20 & 3 & 7 & $91.67 \pm 1.98$ \\
\hline E9 & 30 & 3 & 20 & 3 & 7 & $94.56 \pm 1.90$ \\
\hline E10 & 40 & 1 & 20 & 3 & 7 & $45.23 \pm 2.00$ \\
\hline E11 & 40 & 2 & 20 & 3 & 7 & $42.1 \pm 2.21$ \\
\hline E12 & 40 & 3 & 20 & 3 & 7 & $45 \pm 2.89$ \\
\hline E13 & 50 & 1 & 20 & 3 & 7 & PPT- \\
\hline E14 & 50 & 2 & 20 & 3 & 7 & PPT- \\
\hline E15 & 50 & 3 & 20 & 3 & 7 & PPT- \\
\hline
\end{tabular}




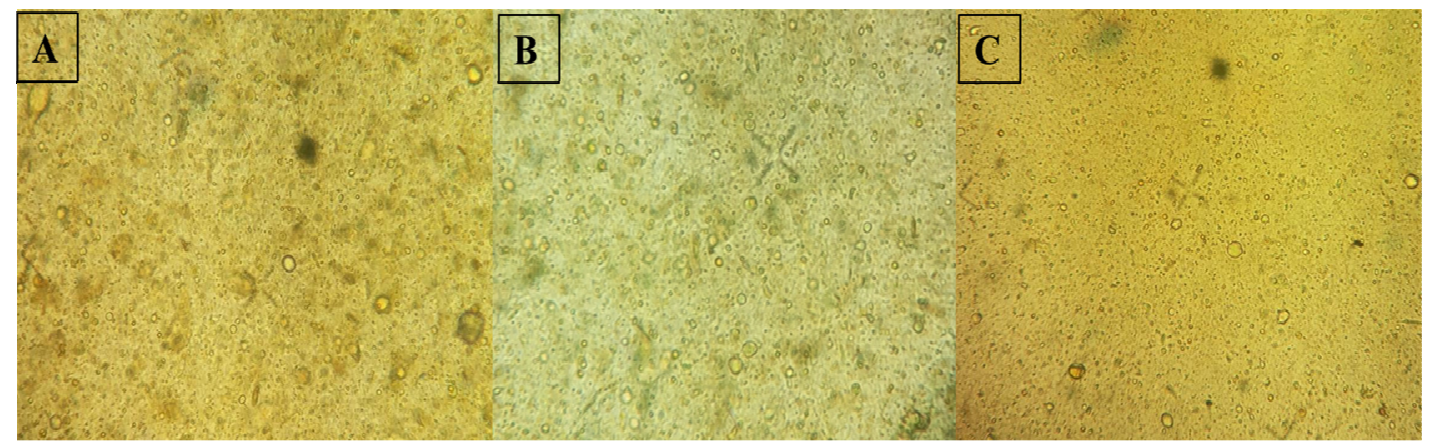

Fig. 3: Photomicrographs of different formulae of CUR loaded ethosomes (A) E9, (B) E 3 and (C) E1. Original magnification x1000.

Table 4: Particle size, polydispersity index, zeta potential and encapsulation efficiency of some CUR ethosome formulations. Each result is the mean value $\pm \operatorname{SD}(n=3)$.

\begin{tabular}{||c|c|c|c|c||}
\hline Formula & $\begin{array}{c}\text { Particle size } \\
(\mathrm{nm})\end{array}$ & PDI & $\begin{array}{c}\text { Zetapotential } \\
(\mathrm{mV})\end{array}$ & EE\% \\
\hline E1 & $182.1 \pm 5.3$ & $0.487 \pm 0.08$ & $-43 \pm 1.6$ & 80 \\
\hline E2 & $201.5 \pm 12.32$ & $0.427 \pm 0.09$ & $-44 \pm 2.6$ & 82 \\
\hline E3 & $262.3 \pm 8.640$ & $0.540 \pm 0.04$ & $-49 \pm 2.6$ & 85 \\
\hline E6 & $301.3 \pm 15.62$ & $0.523 \pm 0.08$ & $-50.5 \pm 1.6$ & 90 \\
\hline E9 & $354.5 \pm 30.1$ & $0.444 \pm 0.11$ & $-64.32 \pm 2.6$ & 93 \\
\hline
\end{tabular}

\section{Encapsulation efficiency of CUR ethosomes}

Table 3 shows the encapsulation efficiency of CUR loaded ethosomes, it is clear that, the CUR EE\% is increased with increasing concentration of lecithin as well as drug loading. Since, increasing lecithin concentration from 1 to $3 \%$ and drug from 10 to $30 \mathrm{mg}$ resulted in increasing of the drug encapsulation efficiency from $80.34 \pm 0.54$ to $94.56 \pm 1.90 \%$ (E1 and E9, respectively). This enhancing effect of lecithin on the drug entrapment efficiency may be due to the hydrophobicity of the drug, which facilitates hydrophobic interactions with the vesicle membranes and thus, enhance drug encapsulation efficiency. Also the increase in the concentration of lecithin may lead to the formation multilayered vesicles which in turn results in improving encapsulation of CUR in the formed layer due to the hydrophobic nature of CUR. These data are in agreement with previous findings ${ }^{35}$.

\section{In-vitro release studies of CUR niosomes and ethosomes}

Figure 4 reveals that, the percent drug release is significantly higher for niosomal formulations $(p<0.05)$ compared to the free CUR. These results confirmed the solubilizing effect of niosomes which gives rise to the improvement of drug release. However this is significant differences in the in-vitro release profiles $(p<0.05)$ may be attributed to other factors such as size of niosomal vesicles and membrane fluidity that depends on alkyl chain length of surfactant ${ }^{36}$. On the other hand, CUR niosome formulation containing (Span 60Tween $60-\mathrm{N} 3)$ exhibited the highest drug release $(85 \%)$, which may be due to the hydrophilic nature of Tween 60, since, as the length of the hydrophilic chain increases; this forms a looser bilayer in the niosomal structure and improves the drug release. The lowest percent of drug release was obtained from niosomes containing GMS (N5), this may be due to the higher transition temperature $\left(65^{\circ} \mathrm{C}\right)$ of GMS. 


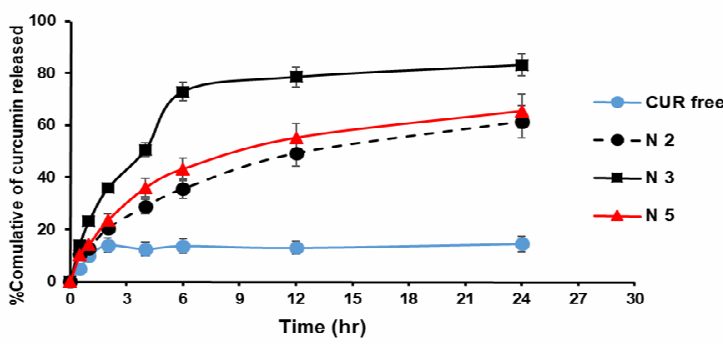

Fig. 4: In-vitro release of noisome formulations containing CUR expressed as percent cumulative drug after $24 \mathrm{hrs}$ in phosphate buffer $\mathrm{pH}$ (5.5). All results are expressed in mean $\pm \operatorname{SD}(n=3)$.

With regard to the release profiles of CUR ethosomes are shown in figure 5. It is obvious that, hydro-alcoholic solution of pure curcumin exhibits significantly higher release rate compared to ethosomal formulations $(p<0.05)$, (most of the drug is released in 2-4 hrs $78.48 \pm 0.712523-92.200 \pm 0.18$, respectively), also it is evident that the release rate of CUR from ethosomes is slower than from hydroalcoholic solution. An initial burst release is observed in the release profile of CUR ethosomes that explained by the diffusion of dissolved drug initially adsorbed on the bilayer surface of the ethosomes ${ }^{37}$. It is noticed that percent of drug release from ethoseme vesicles increased by increasing drug loading from 10 to $30 \mathrm{mg}$ (E3 and E9) and decreasing concentration of lecithin.

Considering the higher EE\% with optimum release profiles from the point of view, CUR niosome N3 and ethosome E3 and E9 were selected for further ex-vivo permeability studies.

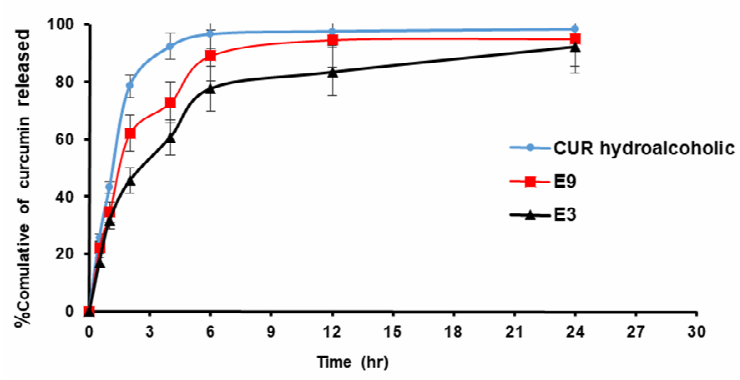

Fig. 5: In-vitro release of ethosome formulations and hydroalcoholic solution containing CUR expressed as percent cumulative drug after $24 \mathrm{hrs}$ in phosphate buffer $\mathrm{pH}$ (5.5). All results are expressed in mean $\pm \mathrm{SD}(n=3)$.

\section{Drug release kinetics}

Release kinetic models are shown in table 5. As this table indicates the correlation coefficient of release data in accordance with Higuchi diffusion release which is higher than other models. In order to better characterize the drug release behavior, Korsmeyer-Peppas model was applied to the linear portion of the curve. The (n) value is found to be $\geq 0.58$ (Table 5) suggesting anomalous (non-Fickian) diffusion. This may indicate that the drug release CUR niosomes and ethosomes is controlled by coupling of diffusion and erosion mechanism.

Table 5: Kinetic analysis of in-vitro release data of CUR from some bilayer vesicles.

\begin{tabular}{|c|c|c|c|c|c|c||}
\hline \multirow{2}{*}{ Formulation } & \multicolumn{2}{|c|}{ Zero order } & \multicolumn{2}{c|}{ Higuchi } & \multicolumn{2}{c|}{ Korsmeyer-Peppas } \\
\cline { 2 - 7 } & $\mathrm{R}^{2}$ & $\mathrm{~K}_{\mathrm{o}}\left(\mathrm{mg} \mathrm{ml}^{-1} \mathrm{hr}^{-1}\right)$ & $\mathrm{R}$ & $\mathrm{K}_{\mathrm{h}}\left(\mathrm{hr}^{-1 / 2}\right)$ & $\mathrm{R}^{2}$ & $\mathrm{n}$ \\
\hline $\mathrm{N} 3$ & 0.9449 & 7.8647 & 0.9895 & 26.27 & 0.989 & 0.58 \\
\hline $\mathrm{N} 5$ & 0.865 & 3.034 & 0.9676 & 15.64 & 0.9790 & 0.58 \\
\hline E3 & 0.885 & 8.931 & 0.979 & 31.51 & 0.996 & 0.74 \\
\hline E9 & 0.834 & 4.76 & 0.984 & 23.67 & 0.983 & 0.60 \\
\hline
\end{tabular}




\section{Ex-vivo skin permeation and penetration studies}

Figures 6 and 7 show the permeability profile of CUR loaded into different niosomes and ethosomes and their gel formulations through rat skin. The cumulative amount of CUR found in the receptor compartment is increased over time for all the tested samples.

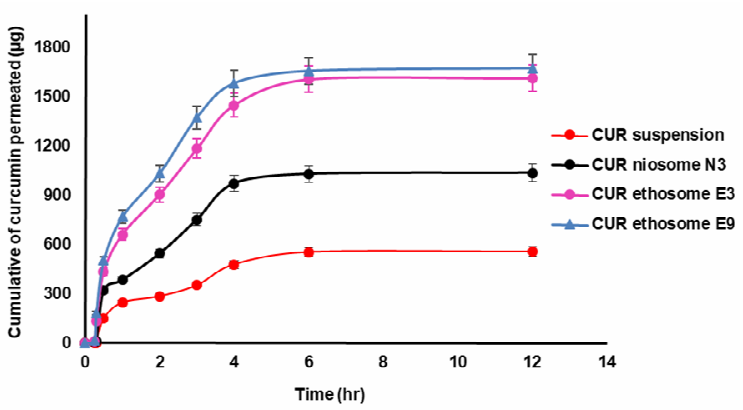

Fig. 6: Cumulative amount of CUR permeated across rat skin CUR (N3), CUR ethosomes (E3 and 9) and CUR suspension. Each value represents the mean $\pm \operatorname{SD}(n=3)$.

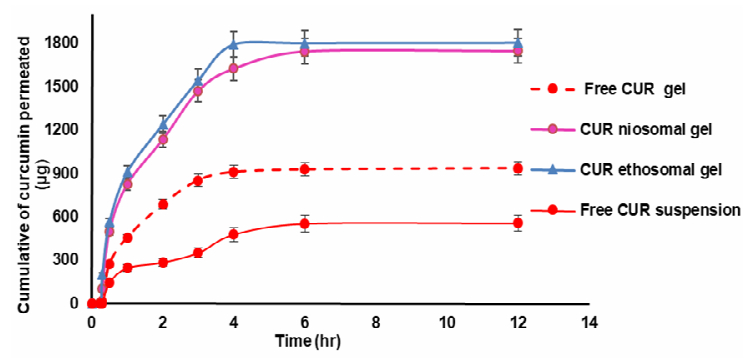

Fig. 7: Cumulative amount of CUR permeated rat skin for, CUR niosomal gel (N3 G), CUR ethosomal gel (E9 G), CUR free gel and CUR free suspension. Each value represents the mean $\pm \operatorname{SD}(n=3)$.

For CUR niosomes, figure 6 shows that the skin permeation of CUR niosomal dispersion (N3) is greater than that from free curcumin suspension. This may be because of the reduction of particle size (nanoscale) and improvement of CUR aqueous solubility which consequently results in enhancing permeability through the skin. On the other hand, the lower permeability of free CUR is due to the penetration from the control curcumin solution which was also influenced by the small amount of propylene glycol used as a co-solvent and not of the pure drug as such, which is completely insoluble in water. The significant higher curcumin flux $(p<0.05)$ that penetrate to the skin is due to the advantages of niosomes in transdermal delivery which can entrap drug inside them and penetrate through the $\operatorname{skin}^{38}$.

In case of CUR ethosomes (Fig. 7), it is noticed that, the cumulative of CUR permeated after $6 \mathrm{hrs}$ increased by a factor of 3.3 and 3.0 for ethosome E9 and E3, respectively when compared to CUR alone.

The improved permeation flux of ethosomes compared with niosomes and control formulations may be attributed to the solvent effect of ethanol on skin lipids followed by skin permeation of ethosomes ${ }^{39}$. When ethosomal formulation comes into contact with skin lipids, ethanol present in the formulations partially fluidizes the intercellular lipids and consequently increases the permeation flux, cumulative drug permeated as well as drug deposition in the $\operatorname{skin}^{40}$. It is also observed that the lag time for all formulations is between 30 to $40 \mathrm{~min}$.

In view of the above results curcumin loaded niosomes (N3) and ethosomes (E9) were selected to incorporate in HPMC 15000 gels $(3 \% \mathrm{w} / \mathrm{v})$.

The permeation profile of CUR of different gel formulations is shown in figure 7. It is observed that CUR permeation into receptor compartment from ethosome and niosome gel formulations is significantly higher than that obtained from free CUR gel $(p<0.05)$. The same observations were previously obtained by Rachmawati et $_{\text {al. }}{ }^{41}$.

Table 6 depicts the permeability coefficient $\left(\mathrm{P}_{\mathrm{app}}\right)$ values of CUR of different formulations, it is clear that, $\left(\mathrm{P}_{\text {app }}\right)$ values of ethosome formulations are significantly higher than that of either CUR niosome formulations or CUR alone $(p<0.05)$. Also table 6 shows that, there is no significant difference between the drug permeability coefficient $\left(\mathrm{P}_{\text {app}}\right)$ of CUR ethosome E9 and E3 $(p<0.05)$.

Furthermore, the permeability coefficient values of CUR ethosome and CUR niosome gel formulations are found to be 3.2, 3.0 times higher than that of CUR free suspension, respectively. In view of this study, the gel containing niosomes (N3) and ethosomes (E9) loaded with CUR were selected to study the in-vivo anti-inflammatory effect of CUR. 
Table 6: Apparent permeability coefficient and permeability enhancement ratio of CUR loaded into different formulations. Each value represents the mean $\pm \mathrm{SD}(n=3)$.

\begin{tabular}{||l|c||}
\hline \multicolumn{1}{|c|}{ Sample } & $\mathrm{P}_{\text {app }}(\mathrm{cm} / \mathrm{sec}) \times 10^{-6}$ \\
\hline CUR free (control) & $2.783 \pm 1.92$ \\
\hline CUR niosome (N3) & $5.321 \pm 3.2$ \\
\hline CUR gel niosome (NG) & $8.364 \pm 1.5$ \\
\hline CUR ethosome (E3) & $7.794 \pm 1.5$ \\
\hline CUR ethosome (E9) & $7.953 \pm 1.3$ \\
\hline CUR ethosme gel (EG) & $8.688 \pm 1.2$ \\
\hline free CUR gel & $4.501 \pm 0.0$ \\
\hline
\end{tabular}

\section{1- Skin retention studies of CUR niosomes and ethosomes gel formulations}

Figure 8 illustrates that CUR niosomes, as well as ethosomes, gives rise to the highest amount of CUR retained or deposited in SC $\left(120.6 \pm 10.31 \mu \mathrm{g} / \mathrm{cm}^{2}\right)$ for niosomes and $\left(80.5 \pm 5.80 \mu \mathrm{g} / \mathrm{cm}^{2}\right)$ for ethosome among other formulations. Such finding revealed that niosomes and ethosomes are suitable for the penetration into the skin and localized as a depot, while gel system of lipid vesicles can deliver more drug to receiving compartment which represents for blood vessels ${ }^{42}$. Therefore, it is worthy to note that, the gel containing niosomes and ethosomes loaded with CUR appeared to be the most appropriate system for a topical anti-inflammatory application. Since they provide improved transdermal penetration and percutaneous absorption of total CUR into the skin.

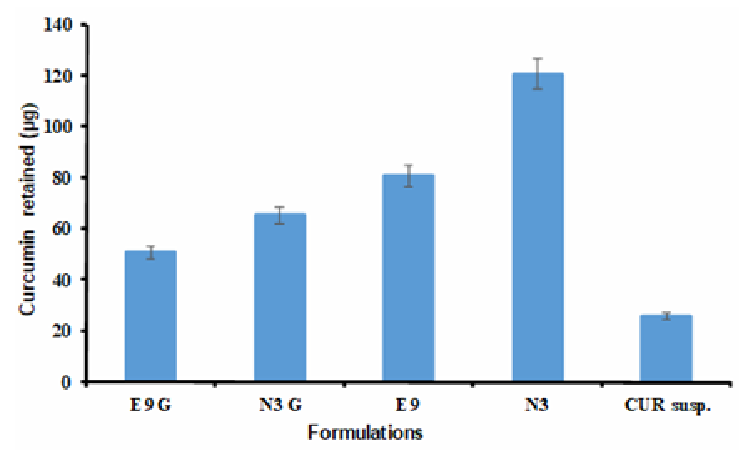

Fig. 8: The Amount of CUR retained in SC after 24 hrs of incubation with rat skin after applying different formulations. Each value represents the mean $\pm \operatorname{SD}(n=3)$.
In-vivo anti-inflammatory effects of different curcumin formulations

Carrageenan-induced paw edema was investigated to evaluate the anti-inflammatory effect of gel formulations containing selected curcumin loaded lipid vesicles (ethosome and niosomes E9, N3). Gel containing free CUR was used as control.

Mean percent inhibition in paw edema of a control group and formulations treated groups of rats is presented in table 7 . In the control group (Group 1), a rapid and continuous increase in paw volume (edema) was observed and the inflammation was sustained during the entire period of $8 \mathrm{hrs}$ of the study. The antiinflammatory activity of curcumin-loaded lipid vesicles gel formulations of both niosome and ethosome was maintained for a longer period. They act as skin-depot forming potential delivery of CUR with high concentration at target site and enhanced permeation of (act as penetration enhancers). In addition, the results in table 7 reveal that there is statistically significant inhibition of paw edema in rats with selected ethosomal and niosomal gel formulations in as compared with free CUR gel $(p<0.05)$. After $3 \mathrm{hrs}$ the percentage inhibition of paw edema by selected ethosome gel $(88.5 \pm 6 \%)$, CUR niosome gel $(66.66 \pm 0.04)$ are approximately three times and two times higher than free CUR $(30.45 \pm 3.5 \%)$, respectively. Above results are supplemented by skin permeation study, which indicates that small vesicle size facilitated CUR to penetrate the stratum corneum (SC) and provide enhanced anti-inflammatory activity. Our observations assumed that the transdermal drug delivery system using ethosomes or niosomes loaded into gel might lead to an expansion in the antiinflammatory use of CUR.

\section{Stability studies}

The optimized formulations (N3, and E9) were selected for stability study based on its invitro performance and stored in tightly closed glass vials at room temperature for 3 months and in the refrigerator $\left(4 \pm 2^{\circ} \mathrm{C}\right)$ for 6 months. As shown in table 8, CUR niosomes show little change in particle size and PDI which indicates stability of the vesicles at these conditions, thus the leakage from the vesicles was minimal. However, the increase in size indicates intervesicular fusion. At room temperature 
Table 7: Anti-inflammatory activity (Percent of inhibition) of different CUR formulation in to male rats. Each value represents the mean $\pm \operatorname{SD}(n=5)$.

\begin{tabular}{||c|c|c|c|c|c||}
\hline Time & $\begin{array}{c}\text { Gr 1 } \\
\text { (Control) }\end{array}$ & $\begin{array}{c}\text { Gr 2 } \\
\text { (nioosmal gel N3) }\end{array}$ & $\begin{array}{c}\text { Gr 3 } \\
\text { (ethosomal gel E9) }\end{array}$ & $\begin{array}{c}\text { Gr 4 } \\
\text { free CUR gel }\end{array}$ & $\begin{array}{c}\text { G5 } \\
\text { Marketed }\end{array}$ \\
\hline 0 & 0 & 0 & 0 & 0 & 0 \\
\hline 1 & 0 & $21.81 \pm 0.01$ & $40 \pm 0.01$ & $9.090 \pm 0.03$ & $36.363 \pm 0.01$ \\
\hline 2 & 0 & $41.66 \pm 0.05$ & $68.333 \pm 0.31$ & $16.666 \pm 0.6$ & $66.666 \pm 0.87$ \\
\hline 3 & 0 & $66.66 \pm 0.04$ & $88.405 \pm 0.04$ & $30.434 \pm 0.81$ & $86.956 \pm 0.41$ \\
\hline 4 & 0 & $71.42 \pm 0.03$ & $92.857 \pm 0.51$ & $38.571 \pm 0.11$ & $90.456 \pm 0.09$ \\
\hline 5 & 0 & $79.45 \pm 0.07$ & $93.150 \pm 0.71$ & $45.205 \pm 0.31$ & $90.410 \pm 0.10$ \\
\hline 6 & 0 & $86.11 \pm 0.08$ & $93.055 \pm 0.51$ & $51.388 \pm 0.61$ & $90.277 \pm 0.34$ \\
\hline 7 & 0 & $86.11 \pm 0.09$ & $93.055 \pm 0.08$ & $51.388 \pm 0.41$ & $93.055 \pm 0.03$ \\
\hline 8 & 0 & $88 \pm 0.018$ & $93.333 \pm 0.08$ & $54.666 \pm 0.21$ & $93.333 \pm 0.03$ \\
\hline
\end{tabular}

Table 8: Characterization of different CUR niosomes and ethosomes after 3 and 6 months.

\begin{tabular}{|c|c|c|c|c||}
\hline Time & \multicolumn{2}{|c|}{3 months } & \multicolumn{2}{c||}{6 months } \\
\hline Formulation & $\mathrm{N} 3$ & $\mathrm{E} 9$ & $\mathrm{~N} 3$ & E9 \\
\hline Size & $424.8 \pm 4.56$ & $524.3 \pm 38.2$ & $524.28 \pm 38.28$ & - \\
\hline PDI & 0.308 & 0.600 & 0.505 & - \\
\hline EE \% & 85 & 76 & 80 & Leakage \\
\hline
\end{tabular}

ethosomes resulted in a slight increase in particle size after 3 months. Some curcumin crystals were observed in ethosomes. The encapsulation efficiency of CUR loaded niosomes maintained stable $(85 \%)$ after 3month storage, compared with ethosome $(76 \%)$ $(p<0.05)$.

The results of the stability experiment showed that CUR niosomes were more rigid than ethosomes under room temperature. This may be due to the presence of high concentration of cholesterol and span 60 in niosomes that increased its rigidity and stability in comparison with ethosome formulations. All the samples were stored in amber-colored glass vials to protect vesicles from oxidation and hydrolysis of the lipids caused by light.

Furthermore, niosomes could protect CUR against degradation as shown in TLC figures (Fig. 9).

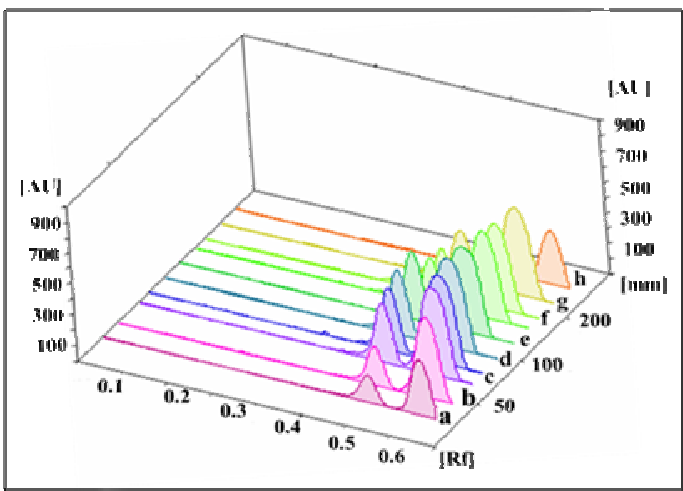

Fig. 9: TLC-scanner 3D diagrams of CUR different formulation subjected to stability studies. CUR- standard (a and b different concentration), CUR niosomes freshly prepared (c), after 3 months (d), CUR ethosome freshly prepared (e), after 3 months (f), CUR niosomes after 6 months at $4^{\circ} \mathrm{C}(\mathrm{g})$ and CUR ethosome after 6 months at $4^{\circ} \mathrm{C}(\mathrm{h})$. 


\section{Thin layer chromatography}

Figure 9, reveals that, 3D chromatograms of free CUR solution have constant characteristic Rf values of $0.57 \mathrm{~mm}$ and 0.48 $\mathrm{mm}$, with nearly the same areas under the peaks. Concentrations of CUR in freshly prepared niosomes are almost similar to that of formulations stored at room temperature for 3 months and no other spots are detected on the chromatograms. This finding indicated the good stability of niosomes. However, it is also noticed that, ethosomes which were stored for 3 months exhibited significantly lower concentration as compared to that of freshly prepared ethosome formulations. This result may be due to the evaporation of ethanol and the presence of a low concentration of cholesterol that leading to leakage of the vesicles and drug precipitation.

\section{Conclusions}

In summary, curcumin was successfully encapsulated in to niosomes and ethosomes with better physicochemical stability for prolonged time period. Selected niosome and ethosome formulations had optimum values of entrapment efficiency, suitable nanosize range for permeation through the rat skin. HPMC15000 gel of selected formulations showed enhanced skin permeation and antiinflammatory activity compared to free drug. The highly stable CUR niosome formulation, exhibited significant cytotoxic effect against Hepatocellular carcinoma cells. Nanosized vesicles (niosome and ethosome) exhibited enhanced biological activity of CUR. They can be considered as promising carriers for transdermal delivery of curcumin and penetration enhancers. In conclusion these results in this work revealed that the formulated bilayer vesicles (ethosome and niosome) are excellent candidates for CUR encapsulation.

\section{REFERENCES}

1- B. B. Aggarwal, A. Kumar and A. C. Bharti, "Anticancer potential of curcumin: Preclinical and clinical studies", Anticancer Research, 23 (1/A), 363-398 (2003).

2- Y. Sun, L. Du, Y. Liu, X. Li, M. Li, Y. Jin and $X$. Qian, "Transdermal delivery of the in- situ hydrogels of curcumin and its inclusion complexes of hydroxypropyl- $\beta$ cyclodextrin for melanoma treatment", Int. J. Pharm., 469 (1), 31-39 (2014).

3- G. Jayaprakasha, L. J. M. Rao and K. Sakariah, "Chemistry and biological activities of C. longa", Trends Food Sci. Technol., 16 (12), 533-548 (2005).

4- M. Tomren, M. Masson, T. Loftsson and H. H. Tønnesen, "Studies on curcumin and curcuminoids: XXXI. Symmetric and asymmetric curcuminoids: Stability, activity and complexation with cyclodextrin", Int. J. of Pharm., 338 (12), 27-34 (2007).

5- B. B. Aggarwal and B. Sung, "Pharmacological basis for the role of curcumin in chronic diseases: An age-old spice with modern targets", Trends Pharmacol. Sci., 30 (2), 85-94 (2009).

6- C. Gong, X. Wei, X. Wang, Y. Wang, G. Guo, Y. Mao, F. Luo and Z. Qian, "Biodegradable self-assembled PEGPCL-PEG micelles for hydrophobic honokiol delivery: I. Preparation and characterization", Nanotechnology, 21 (21), 215103 (2010).

7- A. Liu, H. Lou, L. Zhao and P. Fan, "Validated LC/MS/MS assay for curcumin and tetrahydrocurcumin in rat plasma and application to pharmacokinetic study of phospholipid complex of curcumin", $J$. Pharmaceut. Biomed., 40 (3), 720-727 (2006).

8- V. Kumar, S. A. Lewis, S. Mutalik, D. B. Shenoy and N. Udupa, "Biodegradable microspheres of curcumin for treatment of inflammation", J. Physiol. Pharmacol., 46 (2), 209-217 (2002).

9- X. Wang, Y. Jiang, Y.-W. Wang, M.-T. Huang, C.-T. Ho and Q. "Huang, enhancing anti-inflammation activity of curcumin through O/W nanoemulsions", Food Chem., 108 (2), 419-424 (2008).

10- G. P. Kumar and P. Rajeshwarrao, "Nonionic surfactant vesicular systems for effective drug delivery - an overview", Acta Pharm. Sin. B., 1 (4), 208-219 (2011).

11- A. Priprem, K. Janpim, S. Nualkaew and P. Mahakunakorn, "Topical niosome gel of Zingiber cassumunar Roxb. extract for anti-inflammatory activity enhanced skin 
permeation and stability of compound D", Aaps Pharmscitech, 17 (3), 631-639 (2016).

12- A. Shahiwala and A. Misra, "Studies in topical application of niosomally entrapped nimesulide", J. Pharm. Pharm. Sci., 5 (3), 220-225 (2002).

13- K. M. El-Say, F. I. Abd-Allah, A. E. Lila, A. E.-S. A. Hassan and A. E. A. Kassem, "Diacerein niosomal gel for topical delivery: Development, in-vitro and invivo assessment", J. Liposome Res., 26 (1), 57-68 (2016).

14- J. S. Yuan, M. Ansari, M. Samaan, and E. J. Acosta, "Linker-based lecithin microemulsions for transdermal delivery of lidocaine", Int. J. Pharm., 349 (1-2), 130-143 (2008).

15- H. H. Tønnesen, "Solubility, chemical and photochemical stability of curcumin in surfactant solutions. Studies of curcumin and curcuminoids, XXVIII", Pharmazie, 57 (12), 820-824 (2002).

16- R. L. Thangapazham, A. Puri, S. Tele, R. Blumenthal and R. K. Maheshwari, "Evaluation of a nanotechnology-based carrier for delivery of curcumin in prostate cancer cells", Int. J. Oncol., 32 (5), 11191123 (2008).

17- J. Shaikh, D. Ankola, V. Beniwal, D. Singh and M. R. Kumar, "Nanoparticle encapsulation improves oral bioavailability of curcumin by at least 9 fold when compared to curcumin administered with piperine as absorption enhancer", Eur. J. Pharm. Sci., 37 (3-4), 223-230 (2009).

18- V. Choudhary, H. Shivakumar and H. Ojha, "Curcumin-loaded liposomes for wound healing: Preparation, optimization, in-vivo skin permeation and bioevaluation", J. Drug Deliv. Sci. Technol., 49, 683-691 (2019).

19- M. Anwar, I. Ahmad, M. H. Warsi, S. Mohapatra, N. Ahmad, S. Akhter, A. Ali and F. J. Ahmad, "Experimental investigation and oral bioavailability enhancement of nano-sized curcumin by using supercritical anti-solvent process", Eur. J. Pharm. Biopharm., 96, 162-172 (2015).

20- W. Tiyaboonchai, W. Tungpradit and P. Plianbangchang, "Formulation and characterization of curcuminoids loaded solid lipid nanoparticles", Int. J. Pharm., 337 (1-2), 299-306 (2007).

21- G. Arzani, A. Haeri, M. Daeihamed, H. Bakhtiari-Kaboutaraki and S. Dadashzadeh, "Niosomal carriers enhance oral bioavailability of carvedilol: Effects of bile salt-enriched vesicles and carrier surface charge", Int. J. Nanomedicine, 10, 4797 (2015).

22- M. El-Badry, G. Fetih, D. Fathalla and F. Shakeel, "Transdermal delivery of meloxicam using niosomal hydrogels: Invitro and pharmacodynamic evaluation", Pharm. Dev. Technol., 20 (7), 820-826 (2015).

23- S. Kamboj, V. Saini and S. Bala, "Formulation and characterization of drug loaded nonionic surfactant vesicles (niosomes) for oral bioavailability enhancement", Sci. World. J., 2014 (2014).

24- J. Y. Fang, S. Y. Yu, P. C. Wu, Y. B. Huang and Y. H. Tsai, "In-vitro skin permeation of estradiol from various proniosome formulations", International Journal of Pharmaceutics, 215 (1-2), 9199 (2001).

25- G. J. Xu and H. Sunada, "Influence of formulation changes on drug release kinetics from hydroxypropyl methylcellulose matrix tablets", Chemical \& Pharmaceutical Bulletin, 43, 483-487 (1995).

26- T. Higuchi, "Mechanism of rate of sustained-action medication. Theoretical analysis of rate of solid drugs dispersed in matrices", Journal of Pharmaceutical Sciences, 52, 1145-1149 (1963).

27- R. L. Ritger and N. S. Peppas, "A simple equation for disposition of solute release 11: Fickian and anomalous release from swellable devices", Journal of Controlled Release, 5, 37-42 (1987).

28- N. A. Charoo, A. A. A. Shamsher, K. Kohli, K. Pillai and Z. Rahman, "Improvement in bioavailability of transdermally applied flurbiprofen using tulsi (Ocimum sanctum) and turpentine oil", Colloids Surf. B Biointerfaces, 65 (2), 300-307 (2008).

29- A. Manosroi, P. Wongtrakul, J. Manosroi, H. Sakai, F. Sugawara, M. Yuasa and M. 
Abe, "Characterization of vesicles prepared with various non-ionic surfactants mixed with cholesterol", ibid., 30 (1-2), 129-138 (2003).

30- S. Taymouri and J. Varshosaz, "Effect of different types of surfactants on the physical properties and stability of carvedilol nano-niosomes", Adv. Biomed. Res., 5 (2016).

31- A. H. Alomrani, M. H. Al-Agamy and M. M. Badran, "In-vitro skin penetration and antimycotic activity of itraconazole loaded niosomes: Various non-ionic surfactants", J. Drug Deliv. Sci. Technol., 28, 37-45 (2015).

32- G. Abdelbary and N. El-gendy, "Niosomeencapsulated gentamicin for ophthalmic controlled delivery", Aaps Pharmscitech, 9 (3), 740-747 (2008).

33- A. Pardakhty, J. Varshosaz and A. Rouholamini, "In-vitro study of polyoxyethylene alkyl ether niosomes for delivery of insulin", Int. J. Pharm., 328 (2), 130-141 (2007).

34- W. Faisal, G. M. Soliman and A. M. Hamdan, "Enhanced skin deposition and delivery of voriconazole using ethosomal preparations", J. Liposome Res., 28 (1), 14-21 (2018).

35- M. K. Chourasia, L. Kang and S. Y. Chan, "Nanosized ethosomes bearing ketoprofen for improved transdermal delivery", Results in Pharma Sciences, 1 (1), 60-67 (2011).

36- N. Weiner, N. Williams, G. Birch, C. Ramachandran, C. Shipman and G. Flynn, "Topical delivery of liposomally encapsulated interferon evaluated in a cutaneous herpes guinea pig model", Antimicrob. Agents Chemother., 33 (8), 1217-1221 (1989).
37- S. A. Iizhar, I. A. Syed, R. Satar and S. A. Ansari, "In-vitro assessment of pharmaceutical potential of ethosomes entrapped with terbinafine hydrochloride", Journal of Advanced Research, 7 (3), 453-461 (2016).

38- R. Muzzalupo, L. Tavano, R. Cassano, S. Trombino, T. Ferrarelli and N. Picci, "A new approach for the evaluation of niosomes as effective transdermal drug delivery systems", Eur. J. Pharm. and Biopharm., 79 (1), 28-35 (2011).

39- E. Touitou, N. Dayan, L. Bergelson, B. Godin and M. Eliaz, Ethosomes-novel vesicular carriers for enhanced delivery: Characterization and skin penetration properties", J. of Control. Rel., 65 (3), 403-418 (2000).

40- P. Verma and K. Pathak, "Nanosized ethanolic vesicles loaded with econazole nitrate for the treatment of deep fungal infections through topical gel formulation", Nanomedicine, 8 (4), 489496 (2012).

41- H. Rachmawati, D. K. Budiputra and R. Mauludin, "Curcumin nanoemulsion for transdermal application: Formulation and evaluation", Drug Dev. Ind. Pharm., 41 (4), 560-566 (2015).

42- W. Ruksiriwanich and P. Jantrawut, "Transdermal Delivery Enhancement of Gel Containing Niosomes Loaded with Volvariella Volvacea Extract", In: The Open Conference Proceedings Journal (2015). 


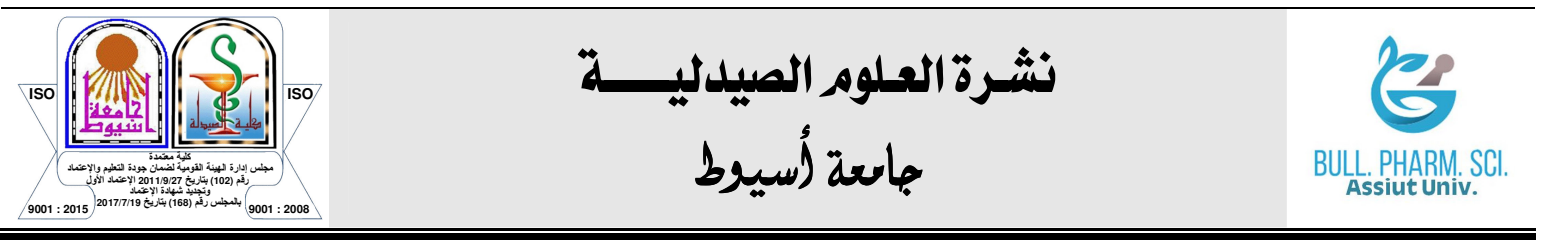

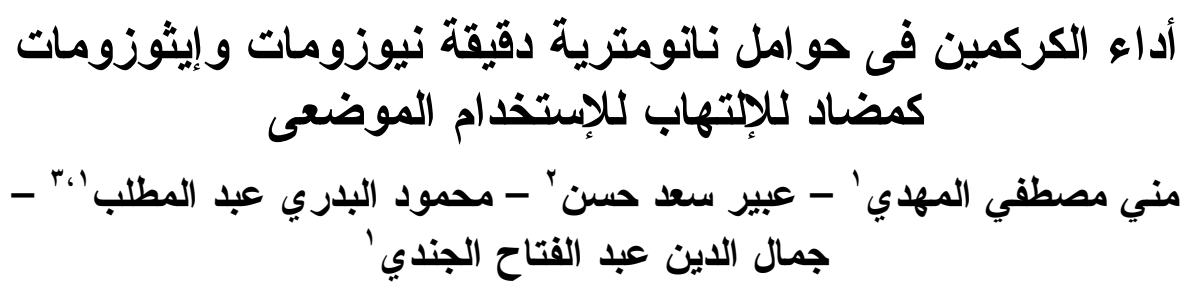

'قسم الصيدلانيات ، كلية الصيدلة ، جامعة أسيوط ، أسيوط ، مصر

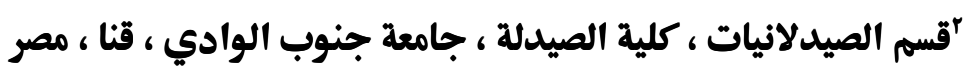

"مركز أسيوط الدولى لأدوية النانو ، مستشفى الراجحى الجامعى للكبد ، جامعة أسيوط ، أسيوط ، مصر ،

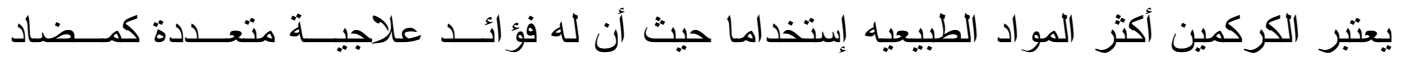

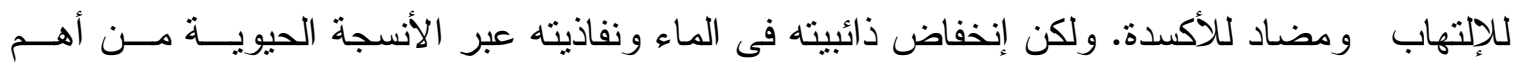

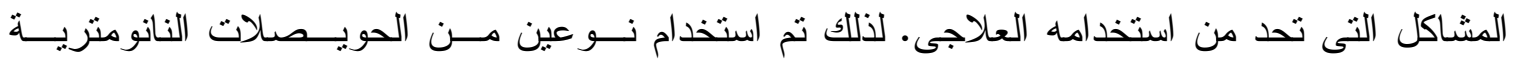

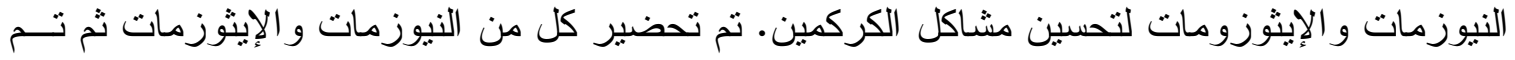

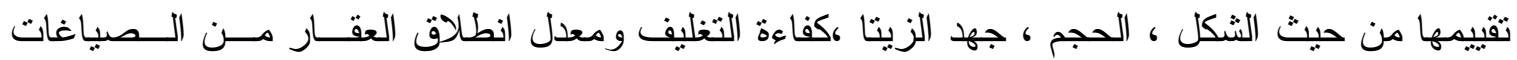
المحضرة. كذلك تم تحميل هذه الصياغات فى بوليمر هيدروكسى بروبيل ميثيل سليلوز HPMC لتكوين

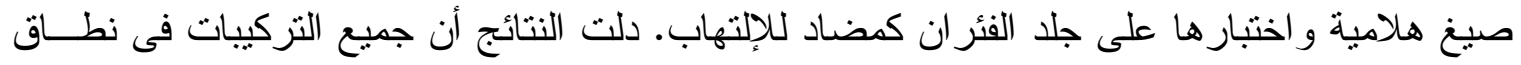

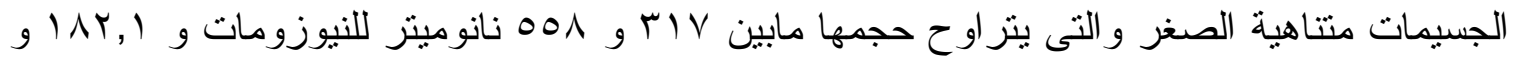

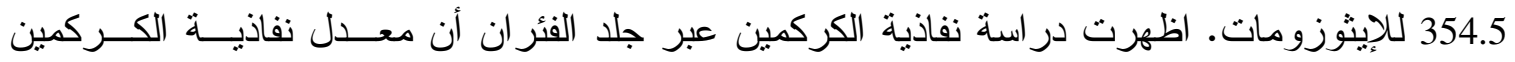

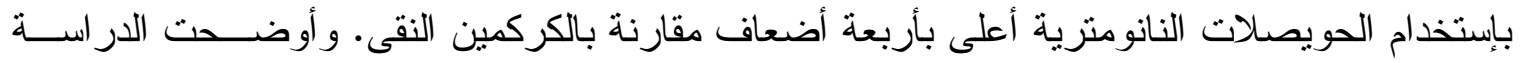

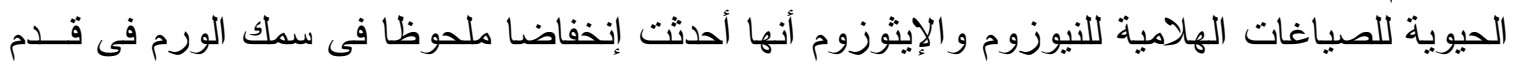
الفئران الناتج عن الكار اجينين مقارنة بالكرمين النقى. 